\title{
The Degree of Autonomic Modulation Is Associated With the Severity of Microvascular Complications in Patients With Type I Diabetes
}

Journal of Diabetes Science and Technology 20I5, Vol. 9(3) 68I-686

(C) 2015 Diabetes Technology Society

Reprints and permissions: sagepub.com/journalsPermissions.nav DOI: 10.1 I $77 / 19322968 \mid 4567226$ dst.sagepub.com

(S)AGE

\author{
Jesper Fleischer, MScBME, PhD', Simon Lebech Cichosz, MSc', \\ Poul Erik Jakobsen, $\mathbf{M D}^{2}$, Knud Yderstraede, $M D^{2}, \mathbf{P h D}^{3}$, \\ Elisabeth Gulichsen, MD ${ }^{4}$, Hans Nygaard, DMSci ${ }^{5}$, \\ Ebbe Eldrup, MD, DMSci ${ }^{6}$, Hans Henrik Lervang, MD, PhD², \\ Lise Tarnow, MD, DMSci ${ }^{4}$, and Niels Ejskjaer, MD, PhD'
}

\begin{abstract}
Objective: The objective of this study was to elucidate whether the degree of autonomic modulation is associated with the degree of microvascular complications in patients with type I diabetes. Methods: A total of 290 type I individuals with diabetes were randomly recruited during normal visits to outpatient clinics at 4 Danish hospitals. The degree of autonomic modulations was quantified by measuring heart rate variability (HRV) during passive spectral analysis and active tests (valsalva ratio [VT], response to standing [RT], and deep breathing [E:I]). To describe possible associations between severity of microvascular complications and measures of autonomic modulation, multivariate analysis was performed. Results: After adjusting for diabetes duration, sex, age, pulse pressure, heart rate, and smoking, autonomic dysfunction remained significantly correlated with severity of retinopathy, nephropathy, and peripheral neuropathy in individuals with type I diabetes patients. Conclusions: Autonomic dysfunction is present in early stages of retinopathy, nephropathy, and peripheral neuropathy in patients with type I diabetes.
\end{abstract}

\section{Keywords}

microvascular complications, type I diabetes, heart rate variability (HRV)

Heart rate variability (HRV) is a widely accepted noninvasive measure of balance/imbalance in the autonomic nervous system, HRV is defined as the variation in heart rate from beat to beat (RR interval) caused by changes in breathing, blood pressure, sympathoadrenal hormones, as well as mental, physical, and certain pathological conditions. In the general population, low HRV has been associated with compromised health and independently predicts all-cause mortality. $^{1-3}$

Moreover, in diabetes patients, the presence of autonomic dysfunction has been suggested as a prognostic marker of complications $^{4,5}$ and the presence of autonomic dysfunction may occur before other late complications. ${ }^{5}$

Detailed HRV analysis, including both cardiovascular reflex tests and frequency domain analysis, are an important tool to estimate cardiac autonomic nervous activity., Several studies indicate that spectral analysis in particular, describing frequency-specific fluctuations in heart rate, may be a more sensitive tool to detect autonomic imbalance compared to active tests. ${ }^{8,9}$ However, it is unknown if the cardiovascular reflex tests and frequency domain analysis decrease according to the severity of microvascular complications or are limited to diagnose cardiovascular autonomic neuropathy (CAN).

The objective of this study was therefore to elucidate the association between the degree of autonomic modulation,

\footnotetext{
'Medical Research Laboratory, Department of Clinical Medicine, Aarhus University and Department of Endocrinology and Internal Medicine, Aarhus University Hospital, Aarhus, Denmark

${ }^{2}$ Department of Endocrinology, Aalborg University Hospital, Aalborg, Denmark

${ }^{3}$ Department of Endocrinology, Odense University Hospital, Odense, Denmark

${ }^{4}$ Steno Diabetes Center, Gentofte, Denmark

${ }^{5}$ Department of Biomedical Engineering, Department of Clinical Medicine, Aarhus University, Aarhus, Denmark

${ }^{6}$ Department of Internal Medicine and Endocrinology, Herlev Hospital, University of Copenhagen, Copenhagen, Denmark

\section{Corresponding Author:}

Jesper Fleischer, Associate Professor, PhD, Aarhus University Hospital, Medical Research Laboratory, Norrebrogade 44, Building 3, 8000 Aarhus C, Denmark.

Email: Jesper.fleischer@clin.au.dk
} 
using both active and passive tests of HRV, with the severity of retinopathy, nephropathy, and peripheral neuropathy.

\section{Methods}

A total of 290 type 1 individuals with diabetes were randomly recruited during normal visits to outpatient clinics at 4 Danish hospitals. The cohort is part of an ongoing study of diabetic autonomic neuropathy. ${ }^{10}$ In brief, over a period of 12 months, patients were recruited consecutively from outpatient clinics at the following hospitals: Aalborg University Hospital, Aarhus University Hospital, Odense University Hospital, and Steno Diabetes Centre. None of the subjects demonstrated alcohol abuse (women 14 units per week and men 21 units per week), congestive heart failure (NYHA classification 3 and 4), arrhythmias preventing HRV calculations, or an existing diagnosis of CAN. The study protocols were approved by the local ethical committee according to the principles of the "Helsinki Declaration II."

\section{Experimental Procedure}

The study was performed as a multicenter cross-sectional observational study. All patients attending the 4 outpatient clinics were a priori eligible for participation and patients who met the inclusion criteria were offered an examination for CAN when appearing in the outpatient clinics. The investigation was logistically planned as an integrated part of the regular visits to the clinics, and the investigation was performed at the end of each visit. Besides testing for CAN, all tests performed in this study reflect the normal clinical procedures in the participating outpatient clinics.

Inclusion criteria included age above 18 years and type 1 diabetes duration of more than 5 years.

Exclusion criteria included alcohol abuse (women more than 14 units per week and men more than 21 units per week), congestive heart failure (NYHA classification 3 and 4), arrhythmias preventing HRV calculations, or an existing diagnosis of CAN.

\section{Experimental Procedure}

Smoking, food, and caffeine-containing liquids were prohibited 2 hours prior to laboratory testing. All laboratory tests (of CAN) were performed between 9 AM and 1 PM in a quiet and isolated examination room. ${ }^{11} \mathrm{HRV}$ and the cardiovascular reflex tests in the 4 centers were measured by technicians using Vagus ${ }^{\mathrm{TM}}$ (Medicus Engineering, Denmark). ${ }^{12,13}$ Blood pressure was measured in a sitting position 2 minutes after examination of CAN. Weight and height were measured and BMI calculated as weight in kilograms divided by the square of height in meters. During the examinations, the individuals with diabetes were questioned for lifestyle habits, for example, smoking and alcohol consumption.

\section{Laboratory Tests}

The following clinical tests for CAN were performed: (1) response to standing (RT), (2) deep breathing (E:I), and (3) valsalva (VT). ${ }^{7,10}$ The heart-rate-based tests were evaluated according to published age-related reference intervals. ${ }^{14}$ CAN was defined using the American Diabetes Association criteria and CAN score was defined as followed: "CAN 0": all tests are normal; "CAN 1": 1 test is abnormal indicating the presence of borderline or early stage CAN; "CAN 2-3": 2 or 3 abnormal tests indicating definite or late stage CAN. ${ }^{15}$ Diabetic peripheral neuropathy (DPN) was examined using either biothesiometri or Semmes-Weinstein monofilament $(10 \mathrm{~g})$ and test results were classified as normal or abnormal. An examination for diabetic retinopathy was performed by an ophthalmologist using a retinal digital camera. Retinopathy was classified as follows (grade 0 to 4 ): normal, mild nonproliferative, moderate nonproliferative, severe nonproliferative or proliferative retinopathy. Nephropathy was classified into 3 groups according to urine albumin-to-creatinine ratio (ACR): normal (ACR $<30$ $\mathrm{mg} / \mathrm{g})$, microalbuminuria $(30 \mathrm{mg} / \mathrm{g} \leq \mathrm{ACR} \leq 300 \mathrm{mg} / \mathrm{g})$, and macroalbuminuria (ACR $>300 \mathrm{mg} / \mathrm{g}$ ). Glycated hemoglobin ( $\mathrm{HbAlc}$ ) was determined by the high-performance liquid chromatography (HPLC) method. Total cholesterol, triglycerides, high-density lipoprotein (HDL), and lowdensity lipoprotein (LDL) cholesterol were measured using an enzymatic method. ${ }^{10}$

\section{HRV in Frequency Domain}

HRV was calculated in time and frequency after 5 minutes of resting in the supine position. To measure the frequencyspecific fluctuations of heart rate during supine resting, we used an autoregressive algorithm ${ }^{16}$ with model order 25 . Three main components are commonly distinguished in a spectrum calculated from an R-R time series: very low frequency (VLF) 0-0.04 Hz, low frequency (LF) 0.04-0.15 $\mathrm{Hz}$, and high frequency (HF) 0.15-0.4 Hz. Thus, VLF represents slower fluctuations caused by regulatory systems and may represent thermoregulatory sympathetic mechanisms. ${ }^{17}$ It is recommended to avoid VLF in short-term recordings (eg, $\leq 5$ minutes) ${ }^{16}$ and therefore, VLF was not considered in this study (due to a recording time of $5 \mathrm{~min}-$ utes). The LF component is influenced by sympathetic, parasympathetic, and baroreflex sensitivity and is characterized by a peak at approximately $0.1 \mathrm{~Hz}$, which may be related to the 10 -second blood pressure waves that reflect oscillations in baroreceptor and chemoreceptor reflex control systems, also known as the Mayer waves. ${ }^{18}$ The high frequency (HF) band from 0.15 to $0.4 \mathrm{~Hz}$ is influenced by parasympathetic sensitivity and by the normal breathing rhythm mainly contributing to power with a center frequency around $0.3 \mathrm{~Hz}{ }^{9}$ 
Table I. Patient Characteristics.

\begin{tabular}{|c|c|}
\hline & Type I \\
\hline$N$ & 290 \\
\hline Females (\%) & 46 \\
\hline Age (years) & $56(9)$ \\
\hline Duration of diabetes (years) & $24(15 ; 37)$ \\
\hline HbAlc (\%) & $8.22(1.36)$ \\
\hline Weight (kg) & $79(16)$ \\
\hline BMI $\left(\mathrm{kg} / \mathrm{m}^{2}\right)$ & $26(5)$ \\
\hline Current smokers (\%) & 23 \\
\hline $\mathrm{DBP}(\mathrm{mmHg})$ & $78(10)$ \\
\hline $\mathrm{SBP}(\mathrm{mmHg})$ & $136(17)$ \\
\hline $\mathrm{PP}(\mathrm{mmHg})$ & $58(16)$ \\
\hline Heart rate (bpm) & $70(12)$ \\
\hline Total cholesterol (mmol/L) & $4.70(1.99)$ \\
\hline LDL cholesterol (mmol/L) & $2.4(0.81)$ \\
\hline HDL cholesterol (mmol/L) & $1.66(0.49)$ \\
\hline Triglycerides (mmol/L) & $0.96(0.70 ; 1.40)$ \\
\hline
\end{tabular}

DBP, diastolic blood pressure; HDL, high density lipoprotein; LDL, low density lipoprotein; OGGT, oral glucose tolerance test; PP, pulse pressure; SBP, systolic blood pressure. Continuous measures are shown as mean (standard deviation) or median (25th; 75 th percentile). Dichotomous variables are shown in percentages.

\section{Statistical Analysis}

Patient characteristics are presented as unadjusted mean (standard deviation) or median (25th, 75th percentile). Transformation with natural logarithms was performed to adjust for skewness and obtain a normal distribution where appropriate. For statistical evaluation and calculation of $P$ values 2-sample $t$ tests and 1-way ANOVA was used for continuous measures, whereas Tukey's least significant difference procedure was used to compare individual differences between measure of autonomic function and severity of complication. To describe possible associations between each HRV parameter and the severity of each complication (retinopathy, nephropathy, and peripheral neuropathy and CAN), ordinal logistic regression analysis was performed.

$P$ values of less than 0.05 were considered statistically significant. MATLAB R2011b (Version 7.13.0.564; MathWorks, Massachusetts, USA), SigmaPlot 11.0 (Systat Software, Inc, California, USA), and Stata 11 (StatCorp LP, Texas, USA) were used for statistical data processing.

\section{Results}

Table 1 shows the baseline characteristics of the group.

Table 2 shows the HRV measures among type 1 diabetes patients by complication - retinopathy, nephropathy, DPNand by complication stage within each category. Table 3 shows the known association between HRV measures and CAN score. There was a significant difference between HRV measures in type 1 diabetes patients with and without complications (ANOVA $P<.05$ ).
Multiple ordinal logistic regression analysis was applied to test possible associations between autonomic dysfunction and complications. After adjusting for sex, age, diabetes duration, smoking, pulse pressure, and heart rate, autonomic dysfunction remained significantly correlated with severity of retinopathy, nephropathy, and peripheral neuropathy in individuals with type 1 diabetes patients (Table 2). In an overall perspective, active tests were predominantly significant, whereas supine resting tests were not. In the retinopathy analysis for nephropathy, patients with no nephropathy differed in all HRV measures from patients with microalbuminuria $(P<.05)$. However, only E:I and VT were able to distinguish between no nephropathy and microalbuminuria. Finally, all HRV measures of patients with CAN score 0 were statistically significantly $(P<.05)$ different from CAN score 1-3.

\section{Discussion}

The main finding of this study is that autonomic dysfunction is present in all stages of microvascular complications. Furthermore, the degree of autonomic modulation is associated with the severity of retinopathy, nephropathy and the presence of peripheral neuropathy in patients with type 1 diabetes. The association remained significant after adjusting for age, diabetes duration, sex, pulse pressure, smoking, and heart rate.

Low HRV has been associated with the presence of symptomatic as well as asymptomatic autonomic dysfunction and is well described in the literature for both type 1 and type 2 diabetes patients. ${ }^{19}$ The finding of autonomic dysfunction in an individual with diabetes is a possible early marker of progression of diabetic complications. The described imbalance in the autonomic nervous system develops early in diabetes ${ }^{20}$ and progresses over time. Furthermore, low HRV is associated with hypertension, ${ }^{21}$ ischemic stroke, ${ }^{22}$ sleep apnoea, ${ }^{6}$ and glycemic variability ${ }^{23,24}$ and may be important in the detection and prevention of hypoglycemia. ${ }^{25,26}$

Spectral analysis of passive HRV testing is used extensively for research purposes and generally believed to supply information about both sympathetic and parasympathetic modulation earlier than other tests. ${ }^{9}$ However, we found that standardized active tests are more reliable to detect autonomic dysfunction related to these complications than are passive HRV tests. The results in Table 2 demonstrate that when adjusting for age, diabetes duration, sex, pulse pressure, smoking, and heart rate only E:I and VT remained associated with the severity of nephropathy, retinopathy, and DPN. Passive testing of HRV was performed in a supine position without controlling breathing rhythm which may be a limitation of this study. Normal spontaneous breathing mainly contributes to energy in the HF band $(0.15-0.4 \mathrm{~Hz})$ with a center frequency around $0.3 \mathrm{~Hz}$ - and the low frequency band remains unaffected by spontaneous breathing. It has been shown the controlled breathing enables easier 
Table 2. Severity of Retinopathy, Nephropathy, and Peripheral Neuropathy and Association to Different HRV Parameters.

\begin{tabular}{|c|c|c|c|c|c|c|c|}
\hline Retinopathy & Grade 0 & Grade I & Grade 2 & Grade 3 & Grade 4 & $P($ ANOVA $)$ & $P$ (multivariate) \\
\hline $\mathrm{N}$ & 100 & 58 & 74 & 32 & 26 & - & - \\
\hline RT (ratio) & $1.19(0.18)$ & $1.18(0.13)$ & $1.14(0.18)$ & $1.12(0.12)$ & $1.09(0.09)$ & .031 & NS \\
\hline E:I (ratio) & $1.24(0.16)$ & $1.22(0.13)$ & $1.15(0.12)$ & $1.09(0.09)$ & $1.06(0.07)$ & .001 & $<.001$ \\
\hline VT (ratio) & $\mathrm{I} .60(0.34)$ & $1.56(0.36)$ & $\mathrm{I} .38(0.24)$ & $1.28(0.22)$ & - & .001 & $<.001$ \\
\hline $\operatorname{TP}\left(\mathrm{ms}^{2}\right)^{\mathrm{a}}$ & $5.63(1.48)$ & $5.65(1.17)$ & $4.74(1.19)$ & $4.53(1.43)$ & $3.97(1.66)$ & .001 & NS \\
\hline $\operatorname{LF}\left(\mathrm{ms}^{2}\right)^{\mathrm{a}}$ & $4.34(1.65)$ & $4.22(1.33)$ & $3.42(1.37)$ & $2.96(1.74)$ & $2.26(2.05)$ & .010 & .006 \\
\hline $\mathrm{HF}\left(\mathrm{ms}^{2}\right)^{\mathrm{a}}$ & $3.78(1.61)$ & $3.86(1.40)$ & $2.95(1.47)$ & $2.53(1.68)$ & $1.98(1.86)$ & .023 & NS \\
\hline Nephropathy & Normal $=0$ & Micro = I & Macro $=2$ & & & & \\
\hline $\mathrm{N}$ & 210 & 66 & 14 & & & - & - \\
\hline RT (ratio) & $1.17(0.16)$ & I.II (0.13) & $\mathrm{I} .07(0.08)$ & & & .002 & NS \\
\hline E:I (ratio) & $1.21(0.15)$ & I.II (0.08) & $1.08(0.07)$ & & & .001 & $<.001$ \\
\hline VT (ratio) & $\mathrm{I} .53(0.33)$ & $1.36(0.28)$ & $1.29(0.23)$ & & & .001 & .016 \\
\hline $\operatorname{TP}\left(\mathrm{ms}^{2}\right)^{\mathrm{a}}$ & $5.39(1.45)$ & $4.53(1.25)$ & $3.97(1.56)$ & & & .003 & .044 \\
\hline $\operatorname{LF}\left(\mathrm{ms}^{2}\right)^{\mathrm{a}}$ & $4.06(1.67)$ & $3.03(1.46)$ & $2.29(1.72)$ & & & .015 & NS \\
\hline $\mathrm{HF}\left(\mathrm{ms}^{2}\right)^{\mathrm{a}}$ & 3.55 (1.67) & $2.65(1.45)$ & $2.29(1.73)$ & & & .007 & NS \\
\hline Peripheral Neuropathy & No $=0$ & Yes $=$ I & & & & & \\
\hline $\mathrm{N}$ & 180 & 110 & & & & - & - \\
\hline RT (ratio) & $1.19(0.16)$ & $1.09(0.12)$ & & & & .001 & .012 \\
\hline E:I (ratio) & $1.22(0.14)$ & $1.12(0.12)$ & & & & .001 & .005 \\
\hline VT (ratio) & $1.56(0.33)$ & $1.32(0.25)$ & & & & .001 & .020 \\
\hline $\operatorname{TP}\left(\mathrm{ms}^{2}\right)^{\mathrm{a}}$ & $5.50(1.35)$ & $4.52(1.47)$ & & & & .002 & NS \\
\hline $\operatorname{LF}\left(\mathrm{ms}^{2}\right)^{\mathrm{a}}$ & $4.19(1.47)$ & $3.00(1.8 I)$ & & & & .047 & NS \\
\hline $\mathrm{HF}\left(\mathrm{ms}^{2}\right)^{\mathrm{a}}$ & $3.65(1.54)$ & $2.68(1.72)$ & & & & .042 & NS \\
\hline
\end{tabular}

Continuous measures are shown as mean (SD). Parametric ANOVA and Tukey's least significant difference procedures were used to compare individual differences between groups. Multiple ordinal logistic regression analysis $P$ (multivariate) with complication severity as a dependent variable was used to adjust for diabetes duration, age, HbAlc, heart rate, and pulse pressure. Retinopathy was classified as follows: normal $=0$, mild $=1$, moderate $=2$, severe $=3$, proliferative $=4$. Nephropathy was classified into 3 groups according to urine albumin-to-creatinine ratio $(A C R): n o r m a l ~(A C R<30 ~ m g / g)$, micro $=$ I $(30 \mathrm{mg} / \mathrm{g} \leq \mathrm{ACR} \leq 300 \mathrm{mg} / \mathrm{g})$, and macro $=2(A C R>300 \mathrm{mg} / \mathrm{g})$. The following HRV parameters were used: valsalva (VT), response to standing $(R T)$, deep breathing (E:I), low frequency power (LF), high frequency power (HF), total power (TP).

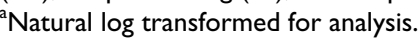

Table 3. Cardiovascular Autonomic Neuropathy (CAN) and Association to Different HRV Parameters.

\begin{tabular}{|c|c|c|c|c|c|c|}
\hline CAN & Score 0 & Score I & Score 2 & Score 3 & $\begin{array}{c}P \\
\text { (ANOVA) }\end{array}$ & $\begin{array}{c}P \\
\text { (multivariate) }\end{array}$ \\
\hline $\mathrm{N}$ & 177 & 54 & 32 & 27 & & \\
\hline RT (ratio) & $1.21(0.16)$ & $1.10(0.10)$ & $1.05(0.14)$ & $1.01(0.01)$ & .001 & $<.001$ \\
\hline E:I (ratio) & $1.24(0.13)$ & $1.14(0.11)$ & $1.04(0.03)$ & $1.03(0.01)$ & .001 & $<.001$ \\
\hline VT (ratio) & $1.62(0.30)$ & $1.32(0.24)$ & $1.21(0.10)$ & I.II (0.04) & .001 & $<.001$ \\
\hline $\mathrm{TP}\left(\mathrm{ms}^{2}\right)^{\mathrm{a}}$ & $5.82(1.15)$ & $4.8 \mathrm{I}(0.98)$ & $3.66(1.13)$ & $3.12(1.15)$ & .001 & .001 \\
\hline $\mathrm{LF}\left(\mathrm{ms}^{2}\right)^{\mathrm{a}}$ & $4.55(1.23)$ & $3.44(1.24)$ & $1.87(1.43)$ & $1.38(1.25)$ & .001 & .002 \\
\hline $\mathrm{HF}\left(\mathrm{ms}^{2}\right)^{\mathrm{a}}$ & $4.04(1.36)$ & $2.77(1.34)$ & $1.55(1.28)$ & $1.56(1.37)$ & .001 & .012 \\
\hline
\end{tabular}

Continuous measures are shown as mean (SD). Parametric ANOVA and Tukey's least significant difference procedures were used to compare individual differences between groups. Multiple ordinal logistic regression analysis $P$ (multivariate) with complication severity as a dependent variable was used to adjust for diabetes duration, age, $\mathrm{HbAlc}$, heart rate, and pulse pressure. CAN score was defined as follows: CAN 0: all tests are normal; CAN I: I test is abnormal indicating the presence of borderline or early stage CAN; CAN 2-3: 2 or 3 abnormal tests indicating definite or late stage CAN. The following HRV parameters were used: valsalva (VT), response to standing (RT), deep breathing (E:I), low frequency power (LF), high frequency power (HF), total power (TP).

${ }^{a}$ Natural log transformed for analysis. separation of the LF and HF bands. ${ }^{6,27}$ However, controlled metronome breathing will also increase the HF band and thereby it may shift the sympathovagal balance toward vagal predominance. ${ }^{28}$

It should be noted that the sample sizes are relatively small in the patient groups with late stage complications. This may account for the finding that some HRV parameters are not significantly different from normal to severe stages of complication severity. Testing for the presence of autonomic dysfunction has been suggested as a prognostic marker of microangiopathic complications $s^{4,5}$ and measurable changes in heart rate may occur before other complications. ${ }^{5}$ Our data support these findings, but larger studies are needed to establish new age-related HRV cutoff values that are not limited to the CAN diagnosis.

In conclusion, this study shows that autonomic dysfunction in individuals with type 1 diabetes is associated with the severity of retinopathy, nephropathy, and peripheral neuropathy. This may indicate that performing proactive tests for 
autonomic dysfunction may be a future risk marker of diabetes complications.

\section{Appendix}

\section{List of Participating Investigators}

Jesper Fleischer, MScBME, $\mathrm{PhD}^{1}$; Simon Lebech Cichosz, $\mathrm{MSc}^{1}$; Poul Erik Jakobsen $\mathrm{MD}^{3}$; Knud Yderstraede, MD, $\mathrm{PhD}^{4}$; Elisabeth Gulichsen $\mathrm{MD}^{5}$; Hans Nygaard, DMSci ${ }^{6}$; Ebbe Eldrup MD $\mathrm{DMSci}^{7}$; Hans Henrik Lervang, MD $\mathrm{PhD}^{3}$; Lise Tarnow, MD DMSci ${ }^{5}$; Niels Ejskjaer, $\mathrm{MD}, \mathrm{PhD}^{1}$

${ }^{1}$ The Medical Research Laboratory, Department of Clinical Medicine, Aarhus University and Department of Endocrinology and Internal Medicine, Aarhus University Hospital, Aarhus, Denmark

${ }^{2}$ Department of Biomedical Sciences, University of Copenhagen, Copenhagen, Denmark

${ }^{3}$ Department of Endocrinology, Aalborg University Hospital, Aalborg, Denmark

${ }^{4}$ Department of Endocrinology, Odense University Hospital, Odense, Denmark

${ }^{5}$ Steno Diabetes Center, Gentofte, Denmark

${ }^{6}$ Department of Biomedical Engineering, Department of Clinical Medicine, Aarhus University, Aarhus, Denmark

${ }^{7}$ Department of Internal Medicine and Endocrinology, Herlev Hospital, University of Copenhagen, Copenhagen, Denmark

\section{Abbreviations}

$\mathrm{ACR}$, albumin-to-creatinine ratio; $\mathrm{BMI}$, body mass index; CAN, cardiovascular autonomic neuropathy; DPN, diabetic peripheral neuropathy; E:I, deep breathing; HbAlc, glycated hemoglobin; HDL, high-density lipoprotein; HF, high frequency; HPLC, highperformance liquid chromatography; HRV, heart rate variability; $\mathrm{LF}$, low frequency; RR, beat to beat interval; RT, response to standing; VLF, very low frequency; VT, valsalva ratio.

\section{Acknowledgments}

Parts of this study were presented in abstract form at the Eleventh Annual Diabetes Technology Meeting, San Francisco, California, October 27-29, 2011. Furthermore, parts of this study were presented in abstract form at the ADA's 2012 Scientific Sessions. The authors thank Ulrick Espelund and Karin Ørbæk Kristensen for valuable comments.

\section{Declaration of Conflicting Interests}

The author(s) declared the following potential conflicts of interest with respect to the research, authorship, and/or publication of this article: JF holds stocks in Medicus Engineering. No other potential conflicts of interest were reported as relevant to this article.

\section{Funding}

The author(s) received no financial support for the research, authorship, and/or publication of this article.

\section{References}

1. Dekker JM, Schouten EG, Klootwijk P, Pool J, Swenne CA, Kromhout D. Heart rate variability from short electrocardiographic recordings predicts mortality from all causes in middle-aged and elderly men. The Zutphen Study. Am J Epidemiol. 1997;145:899-908.

2. Dekker JM, Crow RS, Folsom AR, et al. Low heart rate variability in a 2-minute rhythm strip predicts risk of coronary heart disease and mortality from several causes: the ARIC Study. Atherosclerosis Risk In Communities. Circulation. 2000;102:1239-1244.

3. Facchini M, Malfatto G, Sala L, et al. Changes of autonomic cardiac profile after a 3-week integrated body weight reduction program in severely obese patients. $J$ Endocrinol Invest. 2003;26(2):138-142.

4. Valensi P, Paries J, Attali JR. Cardiac autonomic neuropathy in diabetic patients: influence of diabetes duration, obesity, and microangiopathic complications - the French multicenter study. Metabolism. 2003;52(7):815-820.

5. Pavy-Le TA, Fontaine S, Tap G, Guidolin B, Senard JM, Hanaire H. Cardiovascular autonomic neuropathy and other complications in type 1 diabetes. Clin Auton Res. 2010;20(3):153-160.

6. Vinik AI, Maser RE, Ziegler D. Autonomic imbalance: prophet of doom or scope for hope? Diabet Med. 2011;28(6):643-651.

7. Spallone V, Ziegler D, Freeman R, et al. Cardiovascular autonomic neuropathy in diabetes: clinical impact, assessment, diagnosis, and management. Diabetes Metab Res. 2011;27(7):639-653.

8. Gandhi RA, Marques JL, Selvarajah D, Emery CJ, Tesfaye S. Painful diabetic neuropathy is associated with greater autonomic dysfunction than painless diabetic neuropathy. Diabetes Care. 2010;33(7):1585-1590.

9. Tesfaye S, Boulton AJ, Dyck PJ, et al. Diabetic neuropathies: update on definitions, diagnostic criteria, estimation of severity, and treatments. Diabetes Care. 2010;33(10):2285-2293.

10. Fleischer J, Yderstraede K, Gulichsen E, et al. Cardiovascular autonomic neuropathy is associated with macrovascular risk factors in type 2 diabetes: new technology used for routine large-scale screening adds new insight [published online ahead of print March 31, 2014]. J Diabetes Sci Technol.

11. Spallone V, Bellavere F, Scionti L, et al. Recommendations for the use of cardiovascular tests in diagnosing diabetic autonomic neuropathy. Nutr Metab Cardiovasc Dis. 2011;21(1):69-78.

12. Fleischer J, Nielsen R, Laugesen E, Nygaard H, Poulsen LP, Ejskjaer N. Self-monitoring of cardiac autonomic function at home is feasible. J Diabetes Sci Technol. 2011;5(1): 107-112.

13. Gulichsen E, Fleischer J, Ejskjaer N, Eldrup E, Tarnow L. Screening for diabetic cardiac autonomic neuropathy using a new handheld device. J Diabetes Sci Technol. 2012;6(4): 965-972.

14. Charles M, Fleischer J, Witte DR, et al. Impact of early detection and treatment of diabetes on the 6-year prevalence of cardiac autonomic neuropathy in people with screen-detected diabetes: ADDITION-Denmark, a cluster-randomised study. Diabetologia. 2013;56(1):101-108.

15. Boulton AJ, Vinik AI, Arezzo JC, et al. Diabetic neuropathies: a statement by the American Diabetes Association. Diabetes Care. 2005;28(4):956-962. 
16. Heart rate variability. Standards of measurement, physiological interpretation, and clinical use. Task Force of the European Society of Cardiology and the North American Society of Pacing and Electrophysiology. Eur Heart J. 1996;17(3): 354-381.

17. Engel G, Beckerman JG, Froelicher VF, et al. Electrocardiographic arrhythmia risk testing. Curr Probl Cardiol. 2004;29(7):365-432.

18. Takalo R, Korhonen I, Majahalme S, Tuomisto M, Turjanmaa V. Circadian profile of low-frequency oscillations in blood pressure and heart rate in hypertension. Am $J$ Hypertens. 1999;12(9 pt 1):874-881.

19. Pop-Busui R. Cardiac autonomic neuropathy in diabetes: a clinical perspective. Diabetes Care. 2010;33(2):434-441.

20. Fleischer J, Charles M, Tarnow L, et al. Paper ECG strips may contain overlooked clinical information (ADDITION Denmark). J Diabetes Sci Technol. 2012;6(1):74-80.

21. Istenes I, Keresztes K, Hermanyi Z, et al. Relationship between autonomic neuropathy and hypertension-are we underestimating the problem? Diabet Med. 2008;25(7): 863-866.

22. Ko SH, Song KH, Park SA, et al. Cardiovascular autonomic dysfunction predicts acute ischaemic stroke in patients with type 2 diabetes mellitus: a 7-year follow-up study. Diabet Med. 2008;25(10):1171-1177.

23. Houssay S, Alvariñas $\mathrm{JH}$, Burlando $\mathrm{G}$, et al. The relation of heart rate variability with glycemic variability in type 1 diabetic patients (abstract). Diabetes. 2011;60(suppl 1):658.

24. Dejgaard TF, Fleischer J, Cichosz SL, et al. Autonomic dysfunction is associated with loss of postprandial glycaemic control in newly diagnosed type 2 diabetes patients (abstract). Diabetologia. 2014;57(suppl 1):460-461.

25. Skladnev VN, Tarnavskii S, McGregor T, Ghevondian N, Gourlay S, Jones TW. Hypoglycemia alarm enhancement using data fusion. J Diabetes Sci Technol. 2010;4(1):34-40.

26. Skladnev VN, Ghevondian N, Tarnavskii S, Paramalingam $\mathrm{N}$, Jones TW. Clinical evaluation of a noninvasive alarm system for nocturnal hypoglycemia. J Diabetes Sci Technol. 2010;4(1):67-74.

27. Aysin B, Aysin E. Effect of respiration in heart rate variability (HRV) analysis. Conf Proc IEEE Eng Med Biol Soc. 2006;1:1776-1779.

28. Montano N, Porta A, Cogliati C, et al. Heart rate variability explored in the frequency domain: a tool to investigate the link between heart and behavior. Neurosci Biobehav Rev. 2009;33(2):71-80. 